\title{
Decentralized Power Control with Two-way Training for Multiple Access
}

\author{
Gajanana G Krishna and Srikrishna Bhashyam \\ Dept. of Electrical Engineering \\ Indian Institute of Technology-Madras \\ Chennai, India \\ gajananagk@iitm.ac.in, skrishna@ee.iitm.ac.in
}

\author{
Ashutosh Sabharwal \\ Dept. of Electrical \& Computer Engineering \\ Rice University \\ Houston, TX 77005 \\ ashu@ rice.edu
}

\begin{abstract}
In this work, we analyze the diversity-multiplexing performance of a MIMO multiple access wireless system with non-cooperating transmitters. Each of the transmitters and receiver use noisy and mismatched versions of the channel estimate to implement decentralized power control. While accounting for the resources consumed in training, we show that with relatively simple power control, regardless of the number of transmitters, we can achieve double the maximum diversity order of a system with no instantaneous channel state information at the transmitters. Intuitively, the gain can be attributed to using temporal degrees of freedom enabled by power control without coding over multiple coherence intervals.
\end{abstract}

\section{INTRODUCTION}

In scenarios where many users attempt to communicate to a single receiver, multiple antenna systems have been employed to increase reliability or the supported data rates. A tradeoff between the two competing objectives when there is no channel state information at the transmitter (CSIT) was proposed as the diversity-multiplexing trade-off for the point-to-point MIMO link [1] and later extended to the multiple access channel (MAC)[2]. For quasi-static fading channels, it is known that the order of diversity is the key to link-level performance. To this end, several forms of feedback have been explored to provide CSIT and improve the diversity-multiplexing trade-off (DMT) in both point-to-point MIMO [3],[4] and MAC [5]. However, none of them account for errors and resource consumption on the feedback channel.

A two-way channel formulation was used by the authors in [6] to study the impact of errors in feedback while fully accounting for all resource usage in forward and feedback channels. The two-way formulation is suitable for systems where the channel is symmetric or SNR-symmetric (same SNR in both forward and reverse direction) on a per-link basis, which is often true in time-division duplex systems. For the case of SIMO channel (single input multiple output), the authors showed that the maximum diversity order can be doubled compared to a system which employs no feedback. The key contribution in [6] was the two-way channel formulation and an analysis which accounts for the mismatch in channel state information at the transmitter and receiver.

In this paper, we extend the model of [6] to a MAC scenario. The emphasis in the current work is on understanding in the multi-user context how mismatch in the knowledge of channel at the transmitters and receiver affects the system performance, and if any of the diversity order gains predicted by perfect feedback [3], [4] are achievable with information mismatch.

With $K$ non-cooperating $m$-antenna transmitters adopting decentralized power control and sending messages to a single antenna at the receiver, we show that we can obtain single user performance and nearly a two-fold improvement at all multiplexing gains. The MISO (Multiple Input Single Output) model is then extended to the general MIMO scenario where we characterize an achievable DMT for low multiplexing gains. In particular, we show that in a MIMO MAC system with $m$ antennas at each of the transmitters and $n$ antennas at the receiver, we can attain a maximum diversity of $2 m n$ regardless of the number of transmitters communicating to the receiver, in contrast to only $m n$ with no CSIT [2]. While analyzing the multi-user DMT for MIMO, we also furnish one possible method to extend [6] to the point-to-point MIMO scenario for low multiplexing gain.

In Section II, we describe the two-way training model and define outage events for the non-coherent receiver with training. Section III contain the derivation of DMT for MISO MAC and and Section IV extends the DMT to MIMO MAC. We conclude in Section V.

\section{System Model}

\section{A. Channel Model}

We consider a multiple access channel with $K$ noncooperating transmitters communicating independent messages to a single receiver. The receiver and each of the transmitters transmit over the same channel in a time-division duplexed (TDD) manner. Each transmitter has an array of $m$ transmit antennas and the receiver has an array of $n$ receive antennas. We refer to such a system as a $K$ transmitter $m \times n$ MIMO multiple access channel. We assume a slow fading scenario where the block length of time over which the channel stays constant equal to $l$ symbols is assumed to be long enough to make outage errors dominate the total error probability for the sytem [1][2], $l-T_{o} \geq K m+n-1$ where $T_{o}$ is the training overhead corresponding to the number of symbols used for training either the receiver or a transmitter.

The channel between transmitter $i$ and the receiver is represented by the $n \times m$ matrix $\mathbf{H}_{i t}$. Likewise the channel 
between the receiver and transmitter $i$ is represented by $\mathbf{H}_{i r}^{T}$ where notation $A^{T}$ is taken to mean the transpose of $A$. We say that $\mathbf{H}_{i t}$ and $\mathbf{H}_{i r}$ are symmetric and assume $\mathbf{H}_{i t}=\mathbf{H}_{i r}=\mathbf{H}_{i}$. In the current work, the proposed protocol requires only $S N R$ symmetry such that the singular values of $\mathbf{H}_{i t}$ and $\mathbf{H}_{i r}$ are the same. We statistically model $\left\{\mathbf{H}_{i}\right\}_{i=1, \ldots, K}$ to be i.i.d. and unit variance zero mean circularly symmetric complex Gaussian (ZMCSCG) entries, the richly scattered Rayleigh fading environment.

The signal from the $K$ transmitters to the receiver is given by

$$
\mathbf{Y}_{r}=\sum_{i=1}^{K} \mathbf{H}_{i} \mathbf{X}_{i t}+\mathbf{N}_{r}
$$

where $\mathbf{X}_{i t} \in \mathbb{C}^{m \times 1}$ is the complex, information bearing symbol from the transmitter $i, \mathbf{Y}_{r} \in \mathbb{C}^{n \times 1}$ is the output vector and $\mathbf{N}_{r} \in \mathbb{C}^{n \times 1}$ is the additive noise. In the training phase, the training signal from the receiver to the $i^{\text {th }}$ user is given by

$$
\mathbf{Y}_{i t}=\mathbf{H}_{i}^{T} \mathbf{X}_{r}+\mathbf{N}_{i t}
$$

where $\mathbf{X}_{r} \in \mathbb{C}^{n \times 1}$ is the signal sent by the receiver (e.g. feedback or training), $\mathbf{Y}_{i t} \in \mathbb{C}^{m \times 1}$ is the corresponding signal received at transmitter $i$ and $\mathbf{N}_{i t} \in \mathbb{C}^{m \times 1}$ is the additive noise. The noise at each of the receive and transmit antennas at each time is i.i.d. unit variance ZMCSCG.

\section{B. Training and Power Control}

We build on the model of two-way training in [6] which allows us to accurately model resource usage and address mismatched information in a tractable fashion. The training symbols, which adhere to the same power constraints as data symbols, are transmitted at a constant power equal to the average power constraint of the transmitter denoted by $\bar{P}$.

Phase One of the training protocol begins with the transmission of $n \tau_{R T}$ training symbols ( $\tau_{R T}$ from each of the receive antennas) from the receiver to the $K$ transmitters. The transmitters form the channel estimates as $\widehat{\mathbf{H}}_{i} \forall i=$ $1,2, \ldots, K$. The error in estimation $\widetilde{\mathbf{H}}_{i}$ is given by

$$
\mathbf{H}_{\mathbf{i}}=\widehat{\mathbf{H}}_{i}+\widetilde{\mathbf{H}}_{i} .
$$

Then each of the $K$ transmitters independently utilizes its estimate $\widehat{\mathbf{H}}_{i}$ to decide the power control $P\left(\widehat{\mathbf{H}}_{i}\right)=\kappa p\left(\widehat{\mathbf{H}}_{i}\right)$ where $\kappa \propto \bar{P}$ and $p\left(\widehat{\mathbf{H}}_{i}\right)$ is dependent on the estimate $\widehat{\mathbf{H}}_{i}$. The receiver does not know the channel estimates $\left\{\widehat{\mathbf{H}}_{i}\right\}_{i=1,2, \ldots, K}$ at the end of Phase One of training.

In Phase Two, the $K$ transmitters take turns sending $m \tau_{T R}$ training symbols to the receiver. For reasons explained in detail in [6], the receiver tries to estimate the power controlled channels $\mathbf{G}_{i}=\sqrt{p\left(\widehat{\mathbf{H}}_{i}\right)} \mathbf{H}_{i}$. The power controlled channel is again split into two parts $\widehat{\mathbf{G}}_{i}$, the estimate of the power controlled channel, and $\widetilde{\mathbf{G}}_{i}$, the estimation error, such that

$$
\mathbf{G}_{i}=\widehat{\mathbf{G}}_{i}+\widetilde{\mathbf{G}}_{i}
$$

The entries of $\widetilde{\mathbf{G}}_{i}$ and $\widetilde{\mathbf{H}}_{i}$ are ZMCSCG with variance

$$
\sigma_{\left\{\widetilde{\mathbf{G}}_{i}, \widetilde{\mathbf{H}}_{i}\right\}}^{2}=\frac{1}{\tau_{\{T R, R T\}} \bar{P}}
$$

meeting the Cramèr-Rao bound. Phase Two of training is followed by $\left(l-n \tau_{R T}-K m \tau_{T R}\right)$ symbols of encoded data.

\section{Outage Definition and DMT}

Making the power control in $\mathbf{X}_{\mathbf{i}}$ explicit, we say that $\mathbf{X}_{i t}=\sqrt{P\left(\widehat{\mathbf{H}}_{i}\right)} \mathbf{S}_{i t}$ where $\mathbf{S}_{i t}$ is the unit power complex signal prior to power control. Let $\mathbf{G}=\left[\mathbf{G}_{1} \mathbf{G}_{2} \cdots \mathbf{G}_{K}\right], \widehat{\mathbf{G}}=$ $\left[\widehat{\mathbf{G}}_{1} \widehat{\mathbf{G}}_{2} \ldots \widehat{\mathbf{G}}_{K}\right]$ and $\mathbf{R}(\bar{P})=\left[R_{1}(\bar{P}) R_{2}(\bar{P}) \ldots R_{K}(\bar{P})\right]$ where $R_{i}(\bar{P})$ is the data rate from the $i^{\text {th }}$ user. Also let $\alpha=\left(l-n \tau_{R T}-K m \tau_{T R}\right) / l$ account for rate loss due to training overhead.

Using the ideas of [7, Section 3,4] for a non-coherent pointto-point MIMO link, we define outage for the multiple access non-coherent receiver with training. By an outage event, it is indicated that the channel is so poor that given an estimate of the channel state at the receiver, the target data rate is not supported at least for a subset of the users.

Definition 2.1: For a multiple access channel with $K$ transmitters, each equipped with $m$ transmit antennas and a receiver with $n$ receive antennas, the outage event is

$$
\mathcal{O} \triangleq \bigcup_{\mathcal{W}} \mathcal{O}_{\mathcal{W}}
$$

The union is taken over all subsets $\mathcal{W} \subseteq\{1,2, \ldots, K\}$, and

$$
\mathcal{O}_{\mathcal{W}} \triangleq\left\{\varepsilon(\widehat{G}): I\left(\mathbf{X}_{\mathcal{W}} ; Y \mid \mathbf{X}_{\mathcal{W}^{c}}, \widehat{\mathbf{G}}=\widehat{G}\right)<\alpha \sum_{i \in \mathcal{W}} R_{i}\right\},
$$

where $\varepsilon(\widehat{G})$ is the event that the power controlled channel for $K$ users $\mathbf{G}$ is estimated as $\widehat{G}, \mathbf{X}_{\mathcal{W}}=\left[\mathbf{X}_{i_{1} t}^{T} \mathbf{X}_{i_{2} t}^{T} \cdots \mathbf{X}_{i_{|\mathcal{W}|} t}^{T}\right]^{T} \in$ $\mathbb{C}^{|\mathcal{W}| m \times 1}$ contains the input signals and fading coefficients respectively corresponding to the users in $\mathcal{W}=$ $\left\{i_{1}, i_{2}, \ldots, i_{|\mathcal{W}|}\right\}$.

In the above definition, the outage event $\mathcal{O}_{\mathcal{W}}$ corresponds to correct decoding of information from users in $\mathcal{W}^{c}$ and loss of information from users in $\mathcal{W}$. When $\mathcal{O}_{\mathcal{W}}$ occurs, the receiver would have decoded $\mathbf{X}_{\mathcal{W}^{c}}$ transmitted by users in $\mathcal{W}^{c}$ correctly. The receiver can without loss of optimality, cancel its contribution from the received signals. Using (4), we can write the resultant channel as

$$
\begin{aligned}
\mathbf{Y}_{\mathcal{W}} & =\mathbf{Y}-\sum_{i \in \mathcal{W}^{c}} \widehat{G}_{i} \mathbf{S}_{i} \\
& =\sum_{i \in \mathcal{W}} \widehat{G}_{i} \mathbf{S}_{i}+\sum_{j=1}^{K} \widetilde{G}_{j} \mathbf{S}_{j}+\mathbf{N}_{r} .
\end{aligned}
$$

In order to capture the asymptotic performance of our system, we analyze the diversity-multiplexing tradeoff (DMT) derived in [1], where diversity, $d$ is

$$
d \equiv-\lim _{\bar{P} \rightarrow \infty} \frac{\log (\Pi(\bar{P}, \mathbf{R}(\bar{P})))}{\log (\bar{P})},
$$


which describes the rate at which $\Pi$, the probability of outage event $\mathcal{O}$ defined in (6) and (7), falls with $\bar{P}$ at large $\bar{P}$ for a particular target rate, $\mathbf{R}(\bar{P})$. It is to be noted that we fix a common diversity order requirement for all the users. Multiplexing is defined as

$$
r_{i} \equiv \lim _{\bar{P} \rightarrow \infty} \frac{R_{i}(\bar{P})}{\log (\bar{P})},
$$

where $r_{i}$ quantifies the dependence of the target rate on the average power constraint. We consider the symmetric case where all users have the same rate requirement. The extension to the general case with varying $r_{i}$ follows on the lines of [2].

From [2], we also see that

$$
\operatorname{Pr}(\mathcal{O}) \doteq \operatorname{Pr}\left(\mathcal{O}_{\mathcal{W}^{*}}\right)
$$

where $\mathcal{W}^{*}$ is the subset of $\{1,2, \ldots, K\}$ with the slowest decay rate of $\operatorname{Pr}\left(\mathcal{O}_{\mathcal{W}}\right)^{1}$.

We consider Gaussian codes to determine an achievable bound for the exponent of outage probability. From [8, Theorem 1], we get

$$
I\left(\mathbf{X}_{\mathcal{W}} ; Y \mid \mathbf{X}_{\mathcal{W}^{c}}, \widehat{\mathbf{G}}=\widehat{G}\right)=\log \operatorname{det}\left(\mathbf{I}_{n}+\frac{\kappa \sum_{i \in \mathcal{W}} \widehat{G}_{i} \widehat{G}_{i}^{\dagger}}{m \sigma_{V}^{2}}\right)
$$

where $\sigma_{V}^{2}=1+\frac{\kappa \operatorname{Tr}\left[\sum_{j=1}^{K} \widetilde{G}_{j} \widetilde{G}_{j}^{\dagger}\right]}{m n}$ and $\mathbf{I}_{n}$ is the $n$ dimensional identity matrix. From (11), and noting that $\left\{\widehat{G}_{i}\right\}$ are independent and identically distributed, the outage probability for event $\mathcal{O}_{\mathcal{W}}$ with $|\mathcal{W}|=s$ is given by

$$
\Pi_{s}^{m, n}=\operatorname{Pr}\left(\log \operatorname{det}\left(\mathbf{I}_{n}+\frac{\kappa \sum_{i=1}^{s} \widehat{G}_{i} \widehat{G}_{i}^{\dagger}}{m \sigma_{V}^{2}}\right)<s r \log \bar{P}\right) .
$$

For the current work, we assume a joint $M L$ detector. When all the users have the same diversity order requirement, a joint $M L$ detector fares just as well as an individual $M L$ detector from the arguments given in [2, Section VII]. We note the following Lemma extending [2] to the non-coherent receiver with training.

Lemma 2.2: The curve $d_{K, m, n}^{\hat{R}}\left(\frac{r}{\alpha^{\prime}}\right)$ for a non-coherent receiver with training and no side information at the transmitters is the DMT derived in [2] shifted by a constant scaling factor $\alpha^{\prime}=\left(l-K m \tau_{T R}\right) / l$ following the arguments of [7].

\section{Characterization FOR MISO MAC}

We first consider the case where $n=1$, a MISO system. Let all the transmitters have an equal multiplexing gain of $r$ where $\sum_{i} R_{i}=K r \log (\bar{P})$.

Theorem 3.1: For the multiple access channel considered in the current work, with a single antenna at the receiver, $n=1$, the achievable DMT is given by

$$
d_{\mathrm{MAC}, m, 1}^{*}(r)= \begin{cases}\left(2-\frac{r}{\alpha}\right) m & 0 \leq r<\frac{\alpha}{K} \\ d_{K, m, n}^{\hat{R}}\left(\frac{r}{\alpha^{\prime}}\right) & \frac{\alpha}{K} \leq r \leq \frac{\alpha^{\prime}}{K}\end{cases}
$$

${ }^{1}$ We use $a(x) \doteq b(x)$ to mean $\lim _{x \rightarrow \infty} \frac{a(x)}{x}=\lim _{x \rightarrow \infty} \frac{b(x)}{x}$. Similar definitions hold for $\dot{\leq}, \dot{\geq}$. where $\alpha=\left(l-\tau_{R T}-K m \tau_{T R}\right) / l$ and $\alpha^{\prime}=\left(l-K m \tau_{T R}\right) / l$ account for the loss of rate due to training and $d_{K, m, 1}^{\hat{R}}\left(\frac{r}{\alpha^{\prime}}\right)$ refers to the DMT for a MAC system with no CSIT and a trained receiver.

Proof: When $n=1$, we adopt the power control motivated by [6]

$$
P\left(\widehat{\mathbf{H}}_{i}\right)=\kappa \frac{1}{\widehat{\gamma}_{i}}
$$

where $\widehat{\gamma}_{i}=\widehat{\mathbf{H}}_{i} \widehat{\mathbf{H}}_{i}^{\dagger}$. It can be shown that the power control algorithm in (14) is stable for $m>1$. Also $\sigma_{V}^{2}=1+\frac{\kappa \widetilde{\zeta}^{\prime}}{m}$ where $\widetilde{\zeta}^{\prime}=\sum_{j=1}^{K} \widetilde{G}_{j} \widetilde{G}_{j}^{\dagger}$. We can therefore reduce (12) to

$$
\Pi_{s}^{m, 1}=\operatorname{Pr}\left(\log \left(1+\frac{\kappa \sum_{i=1}^{s} \widehat{\zeta}_{i}}{m+\kappa \widetilde{\zeta}^{\prime}}\right)<\frac{s r}{\alpha} \log \bar{P}\right),
$$

where $\widehat{\zeta}_{i}=\widehat{G}_{i} \widehat{G}_{i}^{\dagger}$. Rearranging (15), we get

$$
\Pi_{s}^{m, 1}=\operatorname{Pr}\left(\kappa \sum_{i=1}^{s} \widehat{\zeta}_{i}<\left(\bar{P}^{\frac{s r}{\alpha}}-1\right)\left(m+\kappa \widetilde{\zeta}^{\prime}\right)\right) .
$$

Putting $s=1$, we obtain the exponent of the outage probability for a single user given in [9]. For $s \geq 1$, we perform the following manipulations.

We bound (16) by the sum of two terms that intuitively separate the effects of uncertainty in the channel state at the transmitters and the receiver. Let $\delta$ be a positive constant.

$$
\begin{aligned}
\Pi_{s}^{m, 1} \leq & \operatorname{Pr}\left(\left(\kappa \sum_{i=1}^{s} \widehat{\zeta}_{i}<P^{\left(\frac{s r}{\alpha}+\delta\right)}\right) \bigcap\left(\kappa \widetilde{\zeta}^{\prime}<m \bar{P}^{\delta}\right)\right) \\
& +\operatorname{Pr}\left(\left(\kappa \sum_{i=1}^{s} \widehat{\zeta}_{i}<P^{\frac{s r}{\alpha}} 2 \kappa \widetilde{\zeta}^{\prime}\right) \bigcap\left(\kappa \widetilde{\zeta}^{\prime} \geq m \bar{P}^{\delta}\right)\right) \\
& \dot{\leq} \underbrace{\operatorname{Pr}\left(\kappa \sum_{i=1}^{s} \widehat{\zeta}_{i}<P^{\left(\frac{s r}{\alpha}+\delta\right)}\right)}_{\Pi_{s 1}^{m, 1}}+\underbrace{\operatorname{Pr}\left(\kappa \widetilde{\zeta}^{\prime}>m \bar{P}^{\delta}\right)}_{\Pi_{s 2}^{m, 1}} \cdot
\end{aligned}
$$

The first term $\Pi_{s 1}^{m, 1}$ arises out of uncertainty in the transmitters' Channel State Information (CSI) while the second term $\Pi_{s 2}^{m, 1}$ comes about from the uncertainty of CSI at the receiver. We notice a slight similarity between (41) in [6] and (17). However, as we shall see, unlike in [6], the partitioning in (17) allows us tackle the multi-user situation better by removing the effect of estimation error at the receiver completely.

Turning our attention to the second term $\Pi_{s 2}^{m, 1}$, we see that

$$
\begin{aligned}
\operatorname{Pr}\left(\kappa \widetilde{\zeta}^{\prime}>m \bar{P}^{\delta}\right) & =\int_{\frac{P^{\delta}}{\kappa}}^{\infty} f_{\widetilde{\zeta}^{\prime}}(x) d x \\
& \stackrel{(a)}{=} \int_{\frac{\bar{P}^{\delta}}{\kappa}}^{\infty} \frac{1}{\Gamma(m) \sigma_{\mathbf{G}}^{2}}\left(\frac{x}{\sigma_{\mathbf{G}}^{2}}\right)^{K m-1} e^{-\frac{x}{\sigma_{G}^{2}}} d x \\
& \stackrel{(b)}{=}\left(\bar{P}^{\delta}\right)^{K m-1} e^{-\bar{P}^{\delta}} .
\end{aligned}
$$

In (a) we have substituted the distribution for the $\chi^{2}$-square variable with $2 \mathrm{Km}$ degrees of freedom and in (b), we have substituted (5). Plugging the exponentially decaying error 
probability of (18) into (8), we see that any $\delta>0$ will yield infinite diversity. The physical implication is that the estimation error at the receiver will not alter the DMT apart from imposing a training overhead. The error in channel state information at the transmitter alone is the limiting factor.

We note that substituting for $G$ in (4), we have

$$
\widehat{G}_{i}=\frac{1}{\sqrt{\widehat{\gamma}_{i}}} H_{i}-\widetilde{G}_{i}=\frac{1}{\sqrt{\widehat{\gamma}_{i}}} \widehat{H}_{i}^{\prime},
$$

where $\widehat{H}_{i}^{\prime}$ is the receiver's normalized estimate of the channel between transmitter $i$ the receiver. Equivalently, using (3), we can write $\widehat{H}_{i}^{\prime}$ in terms of $\widehat{H}_{i}$, the estimate of the channel at the transmitter

$$
\begin{aligned}
\widehat{H}_{i}^{\prime} & =H_{i}-\sqrt{\widehat{\gamma}_{i}} \widetilde{G}_{i} \\
& =\widehat{H}_{i}-\widetilde{H}_{i}^{\prime},
\end{aligned}
$$

where $\widetilde{H}_{i}^{\prime}=\sqrt{\widehat{\gamma}_{i}} \widetilde{G}_{i}-\widetilde{H}_{i}$ is another noise matrix with ZMCSCG entries and variance

$$
\sigma_{\widetilde{\mathbf{H}}_{i}^{\prime}}^{2}=\frac{1}{\tau_{R T} \bar{P}}+\frac{\widehat{\gamma}_{i}}{\tau_{T R} \bar{P}} \doteq(\bar{P})^{-1} .
$$

In essence, the estimate of the channel state at each transmitter is a noisy version of the normalized estimate of the channel state at the receiver with the mismatch given by $\sigma_{\widetilde{\mathbf{H}}_{i}^{\prime}}^{2}$.

Let $\gamma_{i}={\widehat{H^{\prime}}}_{i}{\widehat{H^{\prime}}}_{i}^{\dagger}$. From the definition of (19), we write $\widehat{\zeta}_{i}=\frac{\gamma_{i}}{\widehat{\gamma}_{i}}$. Noting that $\kappa \propto \bar{P}$, the first term, $\Pi_{1 s}^{m, 1}$, in (17) can be recast as

$$
\begin{aligned}
\Pi_{1 s}^{m, 1} & \doteq \operatorname{Pr}\left(\sum_{i=1}^{s} \bar{P}^{\left(1-\frac{s r}{\alpha}-\delta\right)} \frac{\gamma_{i}}{\widehat{\gamma}_{i}}<1\right) \\
& \stackrel{(c)}{\leq} \prod_{i=1}^{s} \operatorname{Pr}\left(\bar{P}^{\left(1-\frac{s r}{\alpha}-\delta\right)} \frac{\gamma_{i}}{\widehat{\gamma}_{i}}<1\right) \\
& \stackrel{(d)}{=} \begin{cases}\left(\bar{P}^{m\left(2-\frac{s r}{\alpha}-\delta\right)}\right) & 0 \leq r<\frac{\alpha(1-\delta)}{s} \\
\bar{P}^{0} & r \geq \frac{\alpha(1-\delta)}{s}\end{cases}
\end{aligned}
$$

where (c) arises out of the independence of $\left\{\frac{\gamma_{i}}{\widehat{\gamma}_{i}}\right\}_{i=1, \ldots, s}$ and (d) comes about from the analysis in [9] using (20) and (21).

Since the analysis of an upper bound to the outage probability is valid for any $\delta>0$, we make $\delta \rightarrow 0^{+}$. Recalling that $\Pi_{s}^{m, 1} \dot{\leq} \Pi_{1 s}^{m, 1}$, we get

$$
\Pi_{s}^{m, 1} \leq \begin{cases}\bar{P}^{m s\left(2-\frac{s r}{\alpha}\right)} & 0 \leq r<\frac{\alpha}{s} \\ \bar{P}^{0} & r \geq \frac{\alpha}{s}\end{cases}
$$

We see from (23) that the DMT curve for error events $\mathcal{O}_{\mathcal{W}}$ with $|\mathcal{W}|>1$ lie above the single user DMT curve up to $r \leq \frac{\alpha}{s}$. Therefore, from (10), the resultant DMT is as given in (13).

Remark 3.2: Unlike the case of no CSIT [2], where the DMT is divided into lightly loaded and heavily loaded regions with single user and $K$ user performances respectively, we get single user performance for almost the full range of permissible multiplexing gain

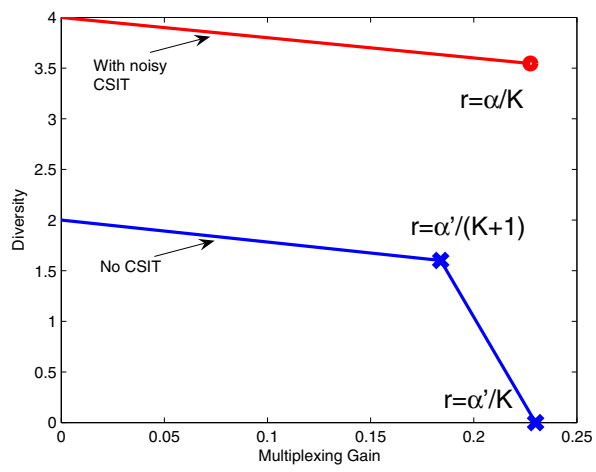

Fig. 1. Achievable DMT of a 4 transmitter $2 \times 1$ MAC system with $\tau_{T R}=$ $\tau_{R T}=10$ symbols and coherence length $l=1000$

We have plotted the DMT for a 4 transmitter $2 \times 1$ multiple access channel in Figure 1 and compared it with the case where there is no side-information at the transmitters.

\section{MIMO MAC FOR LOW MULTIPLEXING GAINS}

We now consider a general MIMO system with $n \geq 1$ and show that at low multiplexing gains, there is a substantial improvement in the DMT for the general scenario as well.

Theorem 4.1: For the multiple access channel considered in the current work, with a general MIMO system, the achievable DMT is given by

$$
d_{\mathrm{MAC}, m, n}^{*}(r)= \begin{cases}\left(2-\frac{r}{\alpha}\right) m n & 0 \leq r<\frac{\alpha}{K} \\ d_{K, m, n}^{\hat{R}}\left(\frac{r}{\alpha^{\prime}}\right) & \frac{\alpha}{K} \leq r \leq \min \left\{m, \frac{n}{K}\right\} \alpha^{\prime}\end{cases}
$$

where $\alpha=\left(l-n \tau_{R T}-K m \tau_{T R}\right) / l$ and $\alpha^{\prime}=\left(l-K m \tau_{T R}\right) / l$ account for the loss of rate due to training and $d_{K, m, n}^{\hat{R}}\left(\frac{r}{\alpha^{\prime}}\right)$ refers to the DMT for a MAC system with no CSIT and a trained receiver.

Proof: Building on the analysis for a MISO MAC system in Section III, we now construct an upper bound for $\Pi_{s}^{m, n}$ for $s=1,2, \ldots, K$. At transmitter $i$, let $\widehat{H}_{i}=$ $\left[\widehat{H}_{i}^{(1) T} \widehat{H}_{i}^{(2) T} \cdots \widehat{H}_{i}^{(n) T}\right]^{T}$, where $\left\{\widehat{H}_{i}^{(j)}\right\}_{j=1}^{n}$ represent the estimates of the $n$ parallel MISO channels from transmitter $i$ to the receiver. Each transmitter implements a worst case power control

$$
P\left(\widehat{\mathbf{H}}_{i}\right)=\kappa \frac{1}{\min _{j} \widehat{\gamma}_{i}^{j}},
$$

where $\widehat{\gamma}_{i}^{j}=\widehat{H}_{i}^{(j)} \widehat{H}_{i}^{(j) \dagger}$. Note that $\kappa \propto \bar{P}$ although different from (14). Let the receiver implement selection and decode the messages at the $n$ antennas separately. As we are operating at low multiplexing gains $(K r<\alpha)$, we do not use the additional degrees of freedom provided by the $n$ antennas and instead use them to improve reliability (diversity). Clearly, the outage event $\mathcal{O}_{\mathcal{W}}$ for such a scheme occurs when all the $n$ MISO links are in outage. After removing the effect of receiver estimation error at each of the the $n$ MISO multiple access 
parallel channels, in the manner of (22), we get

$$
\begin{aligned}
\Pi_{s}^{m, n} & =\prod_{l=1}^{n} \operatorname{Pr}\left(\sum_{i=1}^{s} \bar{P}^{\left(1-\frac{s r}{\alpha}-\delta\right)} \frac{\gamma_{i}^{l}}{\min _{j} \widehat{\gamma}_{i}^{j}}<1\right) \\
& \leq \prod_{l=1}^{n} \operatorname{Pr}\left(\sum_{i=1}^{s} \bar{P}^{\left(1-\frac{s r}{\alpha}-\delta\right)} \frac{\gamma_{i}^{l}}{\widehat{\gamma}_{i}^{l}}<1\right) \\
& \stackrel{(e)}{\leq}\left(\Pi_{s}^{m, 1}\right)^{n} \\
& \doteq \begin{cases}\bar{P}^{s m n\left(2-\frac{s r}{\alpha}-\delta\right)} & 0 \leq r<\frac{(1-\delta) \alpha}{s} \\
\bar{P}^{0} & r \geq \frac{(1-\delta) \alpha}{s}\end{cases}
\end{aligned}
$$

where in (e) we note that the factors in the product are identically distributed multiple access MISO for $l=1,2, \ldots, n$. Setting $\delta \rightarrow 0^{+}$, we again see that the DMT curve for error events $\mathcal{O}_{\mathcal{W}}$ with $s=|\mathcal{W}|>1$ lie above the single user DMT curve up to $r \leq \frac{\alpha}{s}$. Therefore, from (10), the resultant DMT is as given in (24). We switch to a CSIR system for $r \geq \frac{\alpha}{K}$.

In deriving the achievable DMT for multi-user MIMO channel, we have also shown a method of extending the results of [6] for a SIMO channel to the single user point-to-point MIMO channel.

Corollary 4.2: The point-to-point MIMO link with the twoway training model will achieve the DMT $d_{\mathrm{MIMO}, m, n}^{*}(r)$ given below.

$$
d_{\mathrm{MIMO}, m, n}^{*}(r)= \begin{cases}m n\left(2-\frac{r}{\alpha}\right) & 0 \leq r<\alpha \\ d_{\mathrm{MIMO}, m, n}^{\hat{R}}\left(\frac{r}{\alpha^{\prime}}\right) & r \geq \alpha\end{cases}
$$

where $d_{\text {MIMO, } m, n}^{\hat{R}}\left(\frac{r}{\alpha^{\prime}}\right)$ is the fundamental DMT for a point-topoint link for a trained receiver [7].

Proof: Put $s=1$ in (26) to get the result.

In Figure 2, we have plotted the DMT for a 4 transmitter $2 \times 2$ MIMO multiple access channel and compared it with the case where there is no side-information at the transmitters.

The benefit of single user performance at low multiplexing gains enables us to approximately double the diversity order in the range $0 \leq r \leq \frac{\alpha}{K}$. Moreover, for any $K$ transmitter $m \times n$ system, at zero multiplexing gain for all transmitters, i.e. for a fixed rates not varied with power, the diversity order is $2 m n$ compared with $m n$ in a CSIR system irrespective of the number of users. Such a gain can be ascribed to using temporal degrees of freedom obtained from power control without having to code over multiple uncorrelated coherence intervals.

Since a multiple access channel cannot outperform a singleuser channel, we see that decentralized power control performs quite well in our model at low multiplexing gains. For higher multiplexing gains, centralized power control seems to become necessary as the bound quickly falls off. Inversion with smaller eigenvalues, second largest, third largest and so on, might extend the range of multiplexing gain where our scheme of decentralized power control is useful. But the analysis is much more difficult.

Sub-optimal receivers like MMSE or successive cancelation require the number of receive antennas to be larger than the

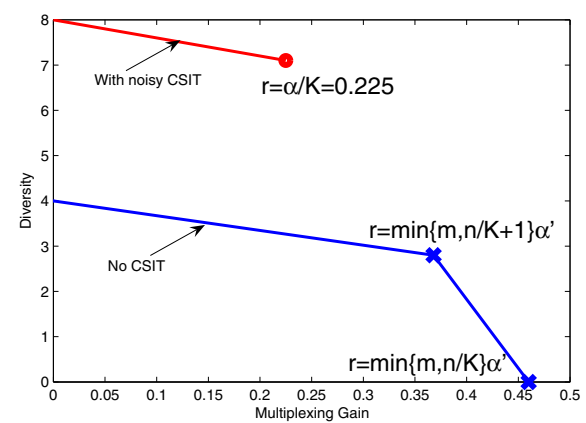

Fig. 2. Achievable DMT of a 4 transmitter $2 \times 2$ system with $\tau_{T R}=$ $\tau_{R T}=10$ symbols and coherence length $l=1000$

number of transmitters, $n>K$. However, as noted in [2], the constraint $n>K$ is not fundamental. We have shown that even with a few antennas at the receiver, we can obtain substantial diversity for an arbitrary number of users over all total multiplexing gains up to unity, $\frac{1}{\alpha} K r<1$, with an optimal joint $M L$ receiver.

\section{CONCLUSION}

In this work, we analyzed multiple access systems using the two-way formulation for feedback channel introduced in [6]. Our results clearly indicate that decentralized power control by non-cooperating transmitters is extremely beneficial at low multiplexing gains even when training the transmitters consumes extra resources and has errors. The maximum diversity in our model with noisy and mismatched channel estimates at the transmitters and the receiver is double that achieved with no side information at the transmitter [2].

\section{REFERENCES}

[1] L. Zheng and D. N. C. Tse, "Diversity and Multiplexing: A Fundamental Tradeoff in Multiple-Antenna Channels," IEEE Trans. Info. Theory, vol. 49, no. 5, pp. 1073-1096, May 2003.

[2] D. N. C. Tse, P. Viswananth, and L. Zheng, " Diversity-multiplexing Tradeoff in Multiple-access Channels," IEEE Trans. Info. Theory, vol. 50, no. 9, pp. 1859- 1874, September 2004.

[3] T. T. Kim and M. Skoglund, "Diversity-multiplexing Tradeoff in MIMO Channels with Partial CSIT," IEEE Trans. Info. Theory, vol. 53, pp. 2743 2759, August 2007.

[4] A. Khoshnevis and A. Sabharwal, "Achievable Diversity and Multiplexing in Multiple Antenna Systems with Quantized Power Control," in Proc. of IEEE International Conference on Communication, Seoul, South Korea, October 2005.

[5] K. N. Lau, Y. Liu, and T. A. Chen, "The Diversity-Multiplexing Tradeoff for the Non-Coherent Multiple Antenna Channel," in Proc. of Allerton Conference, Allerton, IL, October 2002.

[6] C. Steger and A. Sabharwal, "Single-Input Two-Way SIMO Channel: Diversity-Multiplexing Tradeoff with Two-Way Training," accepted for publication in IEEE Trans. Wireless Comm., June 2007.

[7] L. Zheng and D. N. C. Tse, "The Diversity-Multiplexing Tradeoff for the Non-Coherent Multiple Antenna Channel," in Proc. of Allerton Conference, Allerton, IL, October 2002.

[8] H. Hassibi and H. Hochwald, "How Much Training is Needed in MultipleAntenna Wireless Links?" IEEE Trans. Info. Theory, vol. 49, no. 4, pp. 951-963, April 2003.

[9] C. Steger, A. Khoshnevis, A. Sabharwal, and B. Aazhang, "The Case for Transmitter Training," in Proc. of IEEE Int. Symp. Inform. Theory, Seattle, Washington, USA, October 2006. 